\title{
Free energy calculations for solid solutions by computer simulations
}

\author{
By W. G. T. KRANENDONK and D. FRENKEL \\ FOM Institute for Atomic and Molecular Physics, PO Box 41883, \\ 1009 DB Amsterdam, The Netherlands
}

(Received 13 July 1990; accepted 3 September 1990)

\begin{abstract}
Two techniques for calculating the free energy in a binary solid solution of hard spheres are presented. Both compute the free energy difference between a monodisperse system and the mixture. A reversible path is constructed from the solid solution to the monodisperse system-in one case by varying the composition and in the other by changing the diameter ratio. The relative merits of the two methods are discussed. We find that the technique in which the diameter ratio is changed appears more efficient. Examples of application of each technique are presented.
\end{abstract}

\section{Introduction}

Efficient numerical techniques for computing the free energy of solids and fluid are a prerequisite to the construction of phase diagrams by computer simulation. Until recently, most work in this direction was concentrated on monodisperse systems. And, although liquid mixtures have been studied by computer simulation since the early 1970s [1], much less attention has been paid to the study of phase equilibria involving solid solutions. In view of the great practical importance of mixtures, there is clearly a need for numerical techniques for computing the free energy of solid solutions. In addition, theoretical interest in solid solutions has also increased in recent years, in particular in the context of density functional theory. One system that has been studied extensively in this context is a binary mixture of hard spheres with different diameters. This motivated us to attempt a computation of the phase diagram of binary hard sphere mixtures by computer simulation. In the present paper we describe two new techniques for estimating the free energy of a bidisperse mixture of arbitrary composition. Computing the free energy of a dense liquid is relatively simple. The excess free energy can be obtained by integrating the (excess) pressure with respect to the volume. For the solid the situation is more complex. A reversible expansion to infinite volume without any constraints is not possible because an irreversible solid-liquid transition takes place.

To solve this problem for a monodisperse system, two alternative approaches have been developed. The oldest is the single occupancy cell (SOC), introduced by Hoover and Ree [2]. The idea behind this method is to prevent the solid from melting on expansion by adding an external field in the form of cells that restrict the motion of the particles at low densities. A second method is the Einstein crystal method, developed by Frenkel and Ladd [3]. In this method the particles are bound to their lattice sites by springs. By reducing the values of the spring constants to zero, one can construct a reversible path between the Einstein crystal and the solid under consideration. 
However, for substitutionally disordered lattices the methods mentioned above are less suited. This is not because they could not be modified to handle mixtures (although such modifications require some care). The principal reason is that these methods estimate the (relatively large) difference in the free energy between the real mixture and its perfect counterpart. In the perfect mixture the interactions are neglected. However, we are actually interested in the small difference between the free energy of the real mixture and that of its ideal counterpart. The latter is simply the linear interpolation of the free energy of the pure substances plus the ideal entropy of mixing [4].

Here we describe two techniques for computing the excess free energy difference between a solid solution and a monodisperse system at the same volume fraction. As the absolute free energy of a monodisperse system can be computed with high accuracy, the present techniques allow us to compute the absolute free energy of an arbitrary solid solution.

We introduce two ways to construct a reversible path between a monodisperse system and a mixture, characterized by a certain mole fraction and diameter ratio. The first method of constructing a reversible path is by varying the composition at a fixed density and diameter ratio. This technique will be discussed in section 2 . In the second method the diameter ratio is changed from the monodisperse system to that of the mixture of interest, at fixed composition and density. A discussion of this method is given in section 3. The two methods are compared in section 4 . In a subsequent paper [5], we apply one of the techniques described below to compute the phase diagram of a binary mixture of hard spheres.

Throughout this paper we define the diameter ratio $\alpha$ as the ratio between the diameters of the small and large particles in a binary mixture $(\alpha<1 \cdot 0)$. A quantitative measure of the composition of a binary mixture is the mole fraction $X$ of the large particles. $\phi$ is defined as the packing fraction and $\rho^{*}$ as the reduced density

$$
\rho^{*} \equiv \rho \sigma^{3}, \quad \phi \equiv \frac{1}{6} \pi \rho^{*},
$$

where $\rho$ is the number density and $\sigma^{3}=X \sigma_{\mathrm{s}}^{3}+(1-X) \sigma_{\mathrm{L}}^{3}$.

\section{Particle swapping}

In the first method (the 'particle swapping' method) a reversible path is constructed by changing the composition at constant volume and temperature. The method is based on Widom's potential distribution theorem [6]. In a slightly different form, it has already been applied to compute activity coefficients [7] and other partial quantities [8] of liquid mixtures.

Here we confine ourselves to a binary mixture of hard spheres. Consider a system with $N_{\mathrm{A}}$ particles of species $\mathrm{A}$ and $N_{\mathrm{B}}$ particles of species $\mathrm{B}$ in a volume $V$ and at a temperature $T$. The difference in excess free energy $\Delta F^{\mathrm{ex}}$ between this system (0) and that of a system (1) consisting of $N_{\mathrm{A}}+1$ particles of species $\mathrm{A}$ and $N_{\mathrm{B}}-1$ particles of species B at the same temperature $T$ and volume $V$ is

$$
\begin{aligned}
\Delta F^{\mathrm{ex}} & =F_{N_{\mathrm{A}}+1, N_{\mathrm{B}}-1}^{\mathrm{ex}}-F_{N_{\mathrm{A}}, N_{\mathrm{B}}}^{\mathrm{ex}} \\
& =-\beta^{-1} \ln \left(\frac{Q_{N_{\mathrm{A}}+1, N_{\mathrm{B}}-1}}{Q_{N_{\mathrm{A}}, N_{\mathrm{B}}}}\right)
\end{aligned}
$$




$$
=-\beta^{-1} \ln \left[\frac{\int_{v} \exp \left(-\beta U_{1}\right) \mathrm{d} r^{N}}{\int_{v} \exp \left(-\beta U_{0}\right) \mathrm{d} r^{N}}\right],
$$

where $N=N_{\mathrm{A}}+N_{\mathrm{B}}$ is the total number of particles and $\beta=\left(k_{\mathrm{B}} T\right)^{-1}$, with $k_{\mathrm{B}}$ the Boltzmann constant, $\boldsymbol{r}^{N}$ represents all degrees of freedom and the integration is over the whole volume.

$U_{0}$, the potential energy of system 0 , is

$$
U_{0}=\sum_{i, j}^{N_{\mathrm{a}}} U_{\mathrm{AA}}(i, j)+\sum_{i, j}^{N_{\mathrm{A}}, N_{\mathrm{B}}} U_{\mathrm{AB}}(i, j)+\sum_{i, j}^{N_{\mathrm{B}}} U_{\mathrm{BB}}(i, j),
$$

while $U_{1}$, the potential energy of system 1 , is

$$
U_{1}=\sum_{i, j}^{N_{\mathrm{A}}+1} U_{\mathrm{AA}}(i, j)+\sum_{i, j}^{N_{\mathrm{A}}+1, N_{\mathrm{B}}-1} U_{\mathrm{AB}}(i, j)+\sum_{i, j}^{N_{\mathrm{B}}-1} U_{\mathrm{BB}}(i, j),
$$

where we have used $(i, j)$ as a shorthand notation for the coordinates of particles $i$ and $j$. Let $c$ be the particle of species B that is replaced by a particle of species $A$. Then we may write the difference between $U_{0}$ and $U_{1}$ as

$$
\begin{aligned}
\Delta U & =U_{1}-U_{0} \\
& =\sum_{i=1}^{N_{\mathrm{A}}} U_{\mathrm{AA}}(c, i)+\sum_{j=1}^{N_{\mathrm{B}}-1} U_{\mathrm{AB}}(c, j)-\sum_{j=1}^{N_{\mathrm{A}}} U_{\mathrm{AB}}(c, j)-\sum_{j=1}^{N_{\mathrm{B}}-1} U_{\mathrm{BB}}(c, j) .
\end{aligned}
$$

Substitution in (2) gives

$$
\begin{aligned}
\Delta F^{\mathrm{ex}} & =-\beta^{-1} \ln \left[\frac{\int_{v} \exp \left[-\beta\left(U_{0}+\Delta U\right)\right] \mathrm{d} r^{N}}{\int_{v} \exp \left(-\beta U_{0}\right) \mathrm{d} r^{N}}\right] \\
& =-\beta^{-1} \ln \langle\exp (-\beta \Delta U)\rangle_{N, V, T},
\end{aligned}
$$

where the angular brackets symbolize the average in the canonical ensemble. Thus the difference in excess free energy can be calculated as the canonical average of the difference in potential energy between a particle of species $A$ and one of species $B$. This average can be obtained most easily, by a virtual trial move, in which a particle of species B is transformed into one of species A ('swapping'). Because during a MC or MD simulation the positions of the particles are continuously updated, the algorithm for virtual replacement can easily be implemented and takes hardly any extra CPU time.

In the case of hard particles the difference in excess free energy is estimated from the probability to replace a small particle by a larger particle, $P_{0 \rightarrow 1}$, under the restriction that this will not cause any overlap. Actually, instead of keeping the volume constant, we performed the simulations at constant packing fraction. In order to keep the packing fraction constant during a particle swap, we had to increase (decrease) the volume proportionally to the increment (decrement) of the volumechange of the particles each time we tried to change a small (large) particle into a large (small) one $P_{1 \rightarrow 0}$. As we used the acceptance ratio method to compute the free energy change induced by a particle swap, we also had to measure the probability of changing a large particle into a small one. The decrement of the volume to keep the packing 
fraction constant could cause overlap again between other particles. These probabilities to replace a small (large) particle by a large (small) particle at constant packing fraction are denoted by $P_{0 \rightarrow 1}\left(P_{1 \rightarrow 0}\right)$. The difference in excess free energy is now given by the ratio of the two probabilities [9]:

$$
\Delta F^{\text {ex }}=\ln \left(\frac{P_{1 \rightarrow 0}}{P_{0 \rightarrow 1}}\right) .
$$

These probabilities depend strongly on the density at which the trial replacements take place and on the diameter ratio of the particles. Equation (7) gives the free energy difference between two systems that only differ from each other to the extent that one small sphere has been changed into a large one at constant packing fraction. Calculating the difference in free energy between a binary system with a mole fraction $X$ of large spheres and a reference system of monodisperse hard spheres at the same packing fraction requires repeated application of (7): one starts with a monodisperse system of (small) hard spheres, followed by simulations of systems with an increasing number of large spheres, i.e. higher mole fraction of large spheres. For example, if the total number of particles is $N$ and the mole fraction $X$ corresponds to a situation where there are $N_{\mathrm{S}}$ small and $N_{\mathrm{L}}$ large particles, we find the free energy difference with respect to the monodisperse reference system by calculating the sum

$$
\Delta F_{X}^{\mathrm{ex}}=\Delta F_{N, 0}^{\mathrm{ex}}+\Delta F_{N-1,1}^{\mathrm{ex}}+\cdots+\Delta F_{N_{\mathrm{S}}+1, N_{\mathrm{L}}-1}^{\mathrm{ex}}
$$

where

$$
\Delta F_{i, j}^{\mathrm{ex}}=F_{i, j}^{\mathrm{ex}}-F_{i-1, j+1}^{\mathrm{ex}} .
$$

This summation displays two serious drawbacks of the method described above. A practical disadvantage is that for each free energy difference $\Delta F_{i, j}^{\mathrm{ex}}$ a separate simulation must be performed. Hence $N$ simulations must be performed for a system of $N$ particles to cover the whole mole fraction range from zero to one. Instead of virtually replacing one small particle by a large one each time, one can try to replace two or more particles each time. However, because the probability of replacing more than one particle is much smaller than that of replacing just one particle, this will introduce a much larger statistical error, leading to a worse estimate of the free energy difference. In practice, one is forced to use quite small systems. Although the systemsize dependence of differences in chemical potentials is smaller than that of the chemical potential itself [7], tractable systems will probably be too small for reliable estimates. An alternative method, more appropriate for large systems, would be to compute $\Delta F_{i, j}^{\mathrm{ex}}$ at a small number of points and fit a smooth function to these data points. $\Delta F^{\text {ex }}(X)$ itself is then obtained by integrating this function from 0 to $X$.

This would also circumvent a second disadvantage of the particle swapping method, namely the accumulation of statistical errors due to the summation of the individual numerical estimates in (8). This statistical error can be reduced somewhat by noting that both $X=0$ and $X=1$ correspond to a monodisperse system at the same packing fraction. Hence the excess free energies at both endpoints of the swapping process are the same. A second estimate can be obtained by considering a second path: one starts at the system of $N_{\mathrm{S}}$ small and $N_{\mathrm{L}}$ large spheres and, by successively replacing small by large particles, relates this system to the monodisperse system of large spheres:

$$
\Delta F^{\mathrm{ex}}=-\left(\Delta F_{N_{\mathrm{S}}, N_{\mathrm{L}}}^{\mathrm{ex}}+\Delta F_{N_{\mathrm{S}}-1, N_{\mathrm{L}}+1}^{\mathrm{ex}}+\cdots+\Delta F_{\mathrm{l}, N-1}^{\mathrm{ex}}\right),
$$


where we have used (9) again. By taking the weighted average of the results of both paths, one could reduce the statistical error.

We tested the direct summation method for two different diameter ratios $\alpha=0.95$ and $0 \cdot 90$. Constant-volume MC was used to generate the configurations for a system of 32 particles. As a starting configuration, we randomly filled the lattice sites of an FCC lattice with small and large spheres. Each estimate of the free energy differences consisted of a MC run, in which 5000 cycles were used to equilibrate the system and another 15000 to average the desired quantities.

The probabilities $P_{0 \rightarrow 1}$ and $P_{1 \rightarrow 0}$ were measured by attempting virtual transformations of each small particle into a large one after every MC cycle. We did not compute block averages to estimate the statistical error in our data. Rather, we used a consistency check that gives an impression of the overall accuracy of our data (see below).

Tables 1 and 2 show the results of a typical set of the particle swapping simulations on a system of 32 hard spheres. Each row in these tables corresponds to a separate simulation. The swapping process was performed at a packing fraction $\phi=0.575$ $\left(\rho^{*}=1.0982\right)$ for a diameter ratio $\alpha=0.95$ and at $\phi=0.56\left(\rho^{*}=1.0695\right)$ for $\alpha=0.90$. Both tables illustrate the features pointed out above. On going from a diameter ratio of 0.95 to 0.90 , the probabilities $P_{0 \rightarrow 1}$ and $P_{1 \rightarrow 0}$ decrease sharply by a factor of approximately four. Although these probabilities are somewhat higher at lower packing fractions, it seems clear the the swapping method will not be very useful for lower diameter ratios. In other words, the particle swapping method is only applicable to unlike particles of rather similar size. Furthermore, the low probabilities for swapping a single pair of particles suggest that it is useless to attempt simultaneous swaps of more than one pair.

Differences in free energy obtained after summation of the data in tables 1 and 2 , averaged over the two paths, defined by (8) and (10), are collected in table 3 . An impression of the accumulation of the statistical error can be obtained by summing all the excess free energy differences, going from $X=0$ to $X=1$. Because this amounts to the transformation of one monodisperse system into another at the same packing fraction, the total sum should be zero. Instead, the residue for $\alpha=0.95$ is equal to -0.0394 , and that for $\alpha=0.90$ is $-0.399(!)$ A comparison of the particle swapping method with the second method that we have used to compute $\Delta F^{\text {ex }}$ is given in section 3 .

Up till now we have considered substitutionally disordered lattices. However, the method should in principle be able to handle ordered crystals also. A practicle problem in that case is that many substitutionally ordered crystals become unstable in the monodisperse limit. For instance, the sodium chloride lattice transforms into the unstable simple cubic (SC) lattice, and the caesium chloride lattice goes over into the unstable body centred cubic (BCC) lattice. Hence there exists no reversible path between an ordered binary crystal and a stable monodisperse one. As the method discussed in section 3 turns out to be much more convenient for estimating free energy differences for substitutionally ordered crystals, we did not attempt to estimate the free energy of such systems using the particle swapping method.

Of course, the particle swapping method can easily be generalized to both anisotropic hard core potentials and continuous potentials [8]. However, it seems to be quite cumbersome to extend the method to mixtures with more than two components. An advantage is its easy implementation in both MD and MC programs at low extra cost in CPU time. However, it is doubtful that this advantage outweights the severe 
Table 1. Results for the swapping method for a diameter ratio $\alpha=0.95$ at a packing fraction $\phi=0.575 . N_{\mathrm{S}}$ is the number of small particles, $N_{\mathrm{L}}$ is the number of large particles, $X$ is the mole fraction of the large spheres, $P_{0 \rightarrow 1}$ and $P_{1 \rightarrow 0}$ are the probabilities of changing from system 0 to 1 and from system 1 to 0 respectively (see text for an exact definition), and $\Delta F^{\mathrm{ex}}$ is the calculated free energy difference.

\begin{tabular}{|c|c|c|c|c|c|}
\hline$N_{\mathrm{S}}$ & $N_{\mathrm{L}}$ & $X$ & $P_{0 \rightarrow 1}$ & $P_{1 \rightarrow 0}$ & $\Delta F^{\mathrm{ex}}$ \\
\hline 1 & 31 & 0.9688 & 0.246 & 0.203 & -0.192 \\
\hline 2 & 30 & 0.9375 & 0.239 & $0 \cdot 201$ & $-0 \cdot 161$ \\
\hline 3 & 29 & 0.9063 & 0.227 & $0 \cdot 196$ & $-0 \cdot 123$ \\
\hline 4 & 28 & 0.8750 & 0.231 & $0 \cdot 188$ & $-0 \cdot 165$ \\
\hline 5 & 27 & $0 \cdot 8438$ & $0 \cdot 226$ & $0 \cdot 196$ & $-0 \cdot 179$ \\
\hline 6 & 26 & 0.8125 & 0.216 & 0.192 & -0.098 \\
\hline 7 & 25 & 0.7812 & 0.219 & $0 \cdot 180$ & $-0 \cdot 129$ \\
\hline 8 & 24 & 0.7500 & $0 \cdot 208$ & $0 \cdot 169$ & $-0 \cdot 144$ \\
\hline 9 & 23 & 0.7188 & $0 \cdot 206$ & $0 \cdot 184$ & $-0 \cdot 199$ \\
\hline 10 & 22 & 0.6875 & $0 \cdot 206$ & $0 \cdot 186$ & $-0 \cdot 113$ \\
\hline 11 & 21 & 0.6563 & $0 \cdot 198$ & $0 \cdot 182$ & -0.062 \\
\hline 12 & 20 & 0.6250 & $0 \cdot 195$ & $0 \cdot 178$ & -0.069 \\
\hline 13 & 19 & 0.5938 & $0 \cdot 187$ & 0.173 & -0.0488 \\
\hline 14 & 18 & 0.5625 & $0 \cdot 182$ & $0 \cdot 166$ & -0.053 \\
\hline 15 & 17 & 0.5313 & $0 \cdot 182$ & $0 \cdot 164$ & -0.097 \\
\hline 16 & 16 & 0.5000 & $0 \cdot 175$ & $0 \cdot 170$ & -0.067 \\
\hline 17 & 15 & 0.4688 & 0.169 & $0 \cdot 165$ & 0.0072 \\
\hline 18 & 14 & 0.4375 & $0 \cdot 166$ & $0 \cdot 167$ & -0.0099 \\
\hline 19 & 13 & 0.4063 & $0 \cdot 165$ & $0 \cdot 167$ & 0.0145 \\
\hline 20 & 12 & 0.3750 & $0 \cdot 159$ & $0 \cdot 163$ & $0 \cdot 050$ \\
\hline 21 & 11 & 0.3438 & $0 \cdot 156$ & $0 \cdot 162$ & 0.047 \\
\hline 22 & 10 & 0.3125 & 0.155 & $0 \cdot 168$ & 0.048 \\
\hline 23 & 9 & 0.2813 & 0.149 & $0 \cdot 157$ & $0 \cdot 121$ \\
\hline 24 & 8 & 0.2500 & $0 \cdot 147$ & $0 \cdot 148$ & 0.0678 \\
\hline 25 & 7 & 0.2188 & $0 \cdot 144$ & $0 \cdot 162$ & 0.027 \\
\hline 26 & 6 & $0 \cdot 1875$ & $0 \cdot 139$ & $0 \cdot 163$ & $0 \cdot 150$ \\
\hline 27 & 5 & $0 \cdot 1563$ & $0 \cdot 137$ & $0 \cdot 159$ & $0 \cdot 176$ \\
\hline 28 & 5 & 0.1250 & $0 \cdot 133$ & $0 \cdot 156$ & $0 \cdot 183$ \\
\hline 29 & 3 & 0.09375 & $0 \cdot 130$ & $0 \cdot 165$ & $0 \cdot 180$ \\
\hline 30 & 2 & 0.06250 & $0 \cdot 128$ & $0 \cdot 163$ & 0.252 \\
\hline 31 & 1 & 0.03125 & $0 \cdot 128$ & $0 \cdot 170$ & 0.241 \\
\hline mono & mono & 0.0000 & $0 \cdot 125$ & $0 \cdot 203$ & 0.307 \\
\hline
\end{tabular}

practical drawbacks of the method, namely that even for small systems one is forced to do a large number of simulations, that the statistical accuracy of the results tends to be poor and, most importantly, that the method only works for mixtures of particles that are not too dissimilar. A reasonable alternative is discussed in the next section.

\section{The derivative of the free energy with respect to the diameter ratio}

Instead of varying the composition, the free energy of a (solid) mixture can also be computed by evaluating the reversible work that is required to change the diameter ratio of the particles from 1 (monodisperse case) to the value of interest. In this method (hereafter called method II) the mole fractions of the species, the packing fraction and the total volume of the system are kept constant. The change in free energy, when varying the diameter ratio under the stated conditions, can be related to the average collisional momentum transfer between the particles per unit time. The 
Table 2. Results for the swapping method for a diameter ratio $\alpha=0.90$ at a packing fraction $\phi=0.56$. see table 2 for notation.

\begin{tabular}{|c|c|c|c|c|c|}
\hline$N_{\mathrm{S}}$ & $N_{\mathrm{L}}$ & $X$ & $P_{0 \rightarrow 1}$ & $P_{1 \rightarrow 0}$ & $\Delta F^{\mathrm{ex}}$ \\
\hline 1 & 31 & 0.9688 & $0 \cdot 102$ & 0.0592 & -0.451 \\
\hline 2 & 30 & 0.9375 & 0.0976 & 0.0558 & -0.500 \\
\hline 3 & 29 & 0.9063 & 0.0882 & 0.0503 & -0.457 \\
\hline 4 & 28 & 0.8750 & 0.0816 & 0.0500 & -0.483 \\
\hline 5 & 27 & 0.8438 & 0.0768 & 0.0467 & -0.430 \\
\hline 6 & 26 & 0.8125 & 0.0714 & 0.0413 & -0.424 \\
\hline 7 & 25 & 0.7812 & 0.0674 & 0.0434 & -0.491 \\
\hline 8 & 24 & 0.7500 & 0.0606 & 0.0351 & -0.332 \\
\hline 9 & 23 & 0.7188 & 0.0567 & 0.0357 & -0.481 \\
\hline 10 & 22 & 0.6875 & 0.0510 & 0.0345 & -0.356 \\
\hline 11 & 21 & 0.6563 & 0.0461 & 0.0333 & -0.289 \\
\hline 12 & 20 & 0.6250 & 0.0417 & 0.0292 & -0.224 \\
\hline 13 & 19 & 0.5938 & 0.0376 & 0.0291 & $-0 \cdot 252$ \\
\hline 14 & 18 & 0.5625 & 0.0362 & 0.0247 & $-0 \cdot 220$ \\
\hline 15 & 17 & 0.5313 & 0.0316 & 0.0203 & $-0 \cdot 245$ \\
\hline 16 & 16 & $0 \cdot 5000$ & 0.0281 & 0.0213 & $-0 \cdot 323$ \\
\hline 17 & 15 & 0.4688 & 0.0268 & 0.0258 & -0.229 \\
\hline 18 & 14 & 0.4375 & 0.0235 & 0.0230 & 0.094 \\
\hline 19 & 13 & 0.4063 & 0.0213 & 0.0192 & 0.077 \\
\hline 20 & 12 & $0 \cdot 3750$ & 0.0195 & 0.0178 & -0.0195 \\
\hline 21 & 11 & 0.3438 & 0.0180 & 0.0172 & -0.0078 \\
\hline 22 & 10 & $0 \cdot 3125$ & 0.0175 & 0.0183 & -0.0180 \\
\hline 23 & 9 & 0.2813 & 0.0153 & 0.0167 & $0 \cdot 183$ \\
\hline 24 & 8 & 0.2500 & 0.0136 & 0.0185 & 0.210 \\
\hline 25 & 7 & $0 \cdot 2188$ & 0.0130 & 0.0179 & $0 \cdot 348$ \\
\hline 26 & 6 & $0 \cdot 1875$ & 0.0123 & 0.0196 & 0.373 \\
\hline 27 & 5 & $0 \cdot 1563$ & 0.0118 & 0.0210 & 0.506 \\
\hline 28 & 5 & $0 \cdot 1250$ & 0.0107 & 0.0197 & 0.669 \\
\hline 29 & 3 & 0.09375 & 0.0109 & $0 \cdot 0236$ & 0.593 \\
\hline 30 & 2 & 0.06250 & 0.0100 & 0.0239 & $0 \cdot 861$ \\
\hline 31 & 1 & 0.03125 & 0.0094 & 0.0250 & 0.928 \\
\hline mono & mono & 0.0000 & 0.0093 & 0.0647 & 0.993 \\
\hline
\end{tabular}

average momentum exchange per unit time is, in fact, the average radial force acting on each particle. When we decrease the diameter ratio under the abovementioned conditions, we decrease the volumes of the small particles and increase the volumes of the large ones. The reversible work $\mathscr{W}$ (i.e. the change in free energy) associated with an infinitesimal diameter ratio change is equal to the excluded-volume changes multiplied by the appropriate 'pressure' on each particle, i.e. the average force per unit

Table 3. Results of the swapping method for $\alpha=0.95$ and 0.90 . The data for $\alpha=0.95$ were calculated at a packing fraction of $\phi=0.575$ and those for 0.90 at $\phi=0.560$.

\begin{tabular}{cccc}
\hline \multicolumn{2}{c}{$\alpha=0.95$} & \multicolumn{2}{c}{$\alpha=0.90$} \\
\cline { 4 - 5 }$X$ & $\Delta\left(\beta F^{\text {ex }}\right) / N$ & & $\Delta\left(\beta F^{\text {ex }}\right) / N$ \\
\hline 0.2037 & 0.0449 & 0.2037 & 0.154 \\
0.3981 & 0.0580 & 0.3981 & 0.182 \\
0.5000 & 0.0588 & 0.5000 & 0.180 \\
0.6019 & 0.0516 & 0.6091 & 0.153 \\
0.7963 & 0.0302 & 0.7963 & 0.0875 \\
\hline
\end{tabular}


of area of each particle. When we change the diameter ratio from $\alpha$ to $\alpha+\Delta \alpha$, the associated free energy change $\Delta F^{\mathrm{ex}}$ is

$$
\begin{aligned}
\Delta F^{\mathrm{ex}}= & F^{\mathrm{ex}}(\alpha+\Delta \alpha)-F^{\mathrm{ex}}(\alpha)=\mathscr{W} \\
= & 2 \pi \sigma_{\mathrm{S}}^{2} N_{\mathrm{S}} \mathscr{P}_{\mathrm{SS}} \Delta \sigma_{\mathrm{S}}+2 \pi \sigma_{\mathrm{LS}}^{2} N_{\mathrm{S}} \mathscr{P}_{\mathrm{SL}} \Delta \sigma_{\mathrm{S}} \\
& +2 \pi \sigma_{\mathrm{LS}}^{2} N_{\mathrm{L}} \mathscr{P}_{\mathrm{LS}} \Delta \sigma_{\mathrm{L}}+2 \pi \sigma_{\mathrm{L}}^{2} \mathscr{P}_{\mathrm{LL}} \Delta \sigma_{\mathrm{L}} \\
= & \frac{1}{2} N_{\mathrm{S}}\left\langle\frac{\Delta P_{\mathrm{S}}}{\Delta t}\right\rangle_{N, V, E} \Delta \sigma_{\mathrm{S}}+\frac{1}{2} N_{\mathrm{L}}\left\langle\frac{\Delta P_{\mathrm{L}}}{\Delta t}\right\rangle_{N, V, E} \Delta \sigma_{\mathrm{L}}
\end{aligned}
$$

where the subscripts $\mathrm{L}$ and $\mathrm{S}$ correspond to the small and large spheres respectively. $\mathscr{P}_{\alpha \beta}$ is the average radial force per area unit per particle on particle of species $\alpha$ due to collisions with particles of species $\beta$ averaged over all particles. The diameters of the particles are denoted by $\sigma_{\mathrm{S}}$ and $\sigma_{\mathrm{L}}$, and $\sigma_{\mathrm{LS}}=\frac{1}{2}\left(\sigma_{\mathrm{S}}+\sigma_{\mathrm{L}}\right) .\left\langle\Delta P_{\mathrm{S}(\mathrm{L})} / \Delta t\right\rangle$ is the averaged radial force exerted on a small (large) particle. The angular brackets denote an average in the canonical ensemble. The derivative of the free energy with respect to the diameter becomes

$$
\left(\frac{\partial F}{\partial \alpha}\right)=\lim _{\delta \alpha \rightarrow 0} N_{\mathrm{S}}\left\langle\frac{\Delta P_{\mathrm{S}}}{\Delta t}\right\rangle_{N, V, E} \frac{\Delta \sigma_{\mathrm{S}}}{\Delta \alpha}+N_{\mathrm{L}}\left\langle\frac{\Delta P_{\mathrm{L}}}{\Delta t}\right\rangle_{N, V, E} \frac{\Delta \sigma_{\mathrm{L}}}{\Delta \alpha} .
$$

To calculate both radial force contributions, we recall that we keep the total volume of particles constant, while changing the diameter ratio. For $\alpha$ and $\alpha+\Delta \alpha$ respectively,

$$
\begin{aligned}
\alpha: & (1-X) \sigma_{\mathrm{S}}^{3}+X \sigma_{\mathrm{L}}^{3}=\sigma_{0}^{3}, \\
\alpha+\Delta \alpha: & (1-X) \lambda^{3} \sigma_{\mathrm{S}}^{3}+X \mu^{3} \sigma_{\mathrm{L}}^{3}=\sigma_{0}^{3},
\end{aligned}
$$

and

$$
\alpha=\frac{\sigma_{\mathrm{S}}}{\sigma_{\mathrm{L}}}, \quad \alpha+\Delta \alpha=\frac{\lambda \sigma_{\mathrm{S}}}{\mu \sigma_{\mathrm{L}}} .
$$

Equation (13) defines the constant 'mean' diameter $\sigma_{0}$. In (15), $\lambda$ and $\mu$ denote respectively the (as yet undetermined) factor by which $\sigma_{\mathrm{S}}$ is increased and the factor by which $\sigma_{\mathrm{L}}$ is increased. Thus

$$
\lim _{\Delta \alpha \rightarrow 0} \frac{\Delta \sigma_{\mathrm{S}}}{\Delta \alpha}=\lim _{\Delta \alpha \rightarrow 0} \frac{\sigma_{\mathrm{S}}(\lambda-1)}{\Delta \alpha}=\lim _{\Delta \alpha \rightarrow 0} \frac{\sigma_{\mathrm{S}}\left(\lambda^{3}-1\right)}{\Delta \alpha\left(\lambda^{2}+\lambda+1\right)} .
$$

$\lambda^{3}-1$ can be obtained by eliminating $\mu^{3}$ from (14), and we find

$$
\lim _{\Delta \alpha \rightarrow 0} \frac{\Delta \sigma_{\mathrm{S}}}{\Delta \alpha}=\frac{\sigma_{\mathrm{S}} X}{\alpha\left[(1-X) \alpha^{3}+X\right]} .
$$

A similar relation holds for $\lim _{\alpha \rightarrow 0}\left(\Delta \sigma_{\mathrm{L}} / \Delta \alpha\right)$. Substituting (17) into (12) and eliminating $\sigma_{\mathrm{S}}$ and $\sigma_{\mathrm{L}}$ in favour of $\sigma_{0}$, we finally end up with

$$
\frac{1}{N} \frac{\partial F}{\partial \alpha}=\frac{\sigma_{0}}{2}\left\{\frac{X(1-X)}{\left[(1-X) \alpha^{3}+X\right]^{4 / 3}}\left\langle\frac{\Delta P_{\mathrm{S}}}{\Delta t}\right\rangle-\frac{X(1-X) \alpha^{2}}{\left[(1-X) \alpha^{3}+X\right]^{4 / 3}}\left\langle\frac{\Delta P_{\mathrm{L}}}{\Delta t}\right\rangle\right\} \text {. }
$$

The total volume of the particles is given by $\frac{1}{6} \pi N \sigma_{0}^{3}$. In our simulations $\sigma_{0}$ is used as the unit of length and is put equal to 1.

A few remarks about (18) are appropriate. In order to calculate the free energy derivative, we require the same quantities as are needed for the computation of the 
pressure, namely the time-averaged radial momentum transfer for the small and large particles. Because these terms are already sampled during each MD run, implementing the calculation of the derivative is trivial and requires no extra time. In principle, the derivative of the free energy could also be calculated from the values of the radial distribution functions at contact $g_{\alpha \beta}\left(\sigma_{\alpha \beta}\right)$, where $\alpha$ and $\beta$ denote the two species in a binary mixture and $\sigma_{\alpha \beta}$ is the closest distance between two particles. In this way the method could be combined with the conventional MC method. However, because of the statistical uncertainty in the necessary extrapolations of $g_{\alpha \beta}(r)$ to the distance of contact, the MD method is preferable to the MC method.

The computation of the derivative of the free energy involves substracting two terms that are of the same order of magnitude. This means that the derivative of the free energy is at least an order of magnitude smaller than the pressure, whereas the absolute error is about the same. Therefore long runs are necessary for accurate results.

Notice that in the monodisperse limit $(\alpha \rightarrow 1)$ the derivative of the free energy goes to zero for every composition. This can easily be understood by noting that the average momentum transfer for all particles is the same in the monodisperse limit. Because the packing fraction is kept constant during a variation of the diameter ratio, the volume change of small particles compensates that of the large particles. As a result both terms cancel in (18).

Because the present method requires only the computation of the momentum transfer between particles in equilibrium, it is applicable over the entire range of diameter ratios, ranging from zero (points and spheres) to one (monodisperse). This is an important advantage over the method discussed in section 2, which depends on the acceptance of a virtual transformation of a small into a large one.

We have applied this method to both substitutionally disordered and ordered lattices. For the disordered crystals we used a FCC lattice, on which the large and small particles were placed randomly, as a starting configuration. The system size was 108 particles. The MD method was used to generate configurations. To sample configuration space more efficiently, we attempted to interchange randomly chosen small and large spheres every 100 collisions. We accepted the interchange if it did not cause overlap with the other particles. The first $2 \cdot 1 \times 10^{5}$ collisions were used to equilibrate the system, followed by $3.9 \times 10^{5}$ collisions to average the desired quantities. The errors in the sampled quantities were estimated by dividing the run into blocks of 15000 collisions, over which the mean and standard deviation of all quantities of interest were calculated.

Table 4 shows some typical results obtained with the present method for a mixed crystal of hard spheres over a range of compositions. The free energy derivative turns out to be always negative for substitutionally disordered crystals, which means that the free energy increases as the spheres become more dissimilar. To illustrate the fact that the free energy derivative has a much larger relative statistical error than the pressure, we also include the corresponding pressure data in the table. The effect of substraction in (18) is obvious: for all diameter ratios the absolute value of the derivative is at least one order of magnitude smaller than that of the pressure. As the absolute errors are approximately the same, the relative error is at least one order larger. This effect is most pronounced for diameter ratios near the monodisperse limit. As was discussed above, in the monodisperse limit the derivative should go to zero. Especially at mole fractions $X$ near 0 or 1, substraction leads to large relative errors. Fortunately, it is the absolute errors that matter, and these are not pathological for $\alpha$ close to 1. 
Table 4. Results of method II (see text) for a substitutionally disordered FCC lattice. The derivative of the free energy with respect to the diameter ratio per particle $N^{-1} \partial F^{\mathrm{ex}} / \partial \alpha$ and the pressure $\beta P \sigma^{3}$ represented as functions of the diameter ratio $\alpha$ and mole fraction $X$ at a packing fraction $\phi=0.545$.

\begin{tabular}{|c|c|c|c|c|c|}
\hline \multicolumn{3}{|c|}{$X=0 \cdot 111$} & \multicolumn{3}{|c|}{$X=0.2037$} \\
\hline$\alpha$ & $N^{-1} \partial F^{\mathrm{ex}} / \partial \alpha$ & $\beta P \sigma^{3}$ & $\alpha$ & $N^{-1} \partial F^{\mathrm{ex}} / \partial \alpha$ & $\beta P \sigma^{3}$ \\
\hline 0.850 & $-2 \cdot 90(23)$ & $13 \cdot 89(13)$ & 0.850 & $-3.63(29)$ & $14 \cdot 79(16)$ \\
\hline 0.867 & $-2 \cdot 585(21)$ & $13 \cdot 357(96)$ & 0.867 & $-3 \cdot 56(45)$ & $14 \cdot 19(23)$ \\
\hline 0.883 & $-2 \cdot 20(11)$ & $12 \cdot 910(58)$ & 0.883 & $-3 \cdot 11(19)$ & $13 \cdot 519(91)$ \\
\hline 0.900 & $-1.781(93)$ & $12 \cdot 528(49)$ & 0.900 & $-2 \cdot 58(12)$ & $12 \cdot 968(45)$ \\
\hline 0.910 & $-1 \cdot 522(88)$ & $12 \cdot 326(50)$ & 0.910 & $-2 \cdot 33(12)$ & $12 \cdot 703(56)$ \\
\hline 0.925 & $-1 \cdot 190(72)$ & $12 \cdot 097(34)$ & 0.925 & $-1 \cdot 818(79)$ & $12 \cdot 347(35)$ \\
\hline 0.950 & $-0.682(60)$ & $11 \cdot 835(32)$ & 0.950 & $-1.099(85)$ & $11.946(31)$ \\
\hline 0.975 & $-0.293(81)$ & $11 \cdot 713(41)$ & 0.975 & -0.475 & $11 \cdot 739(24)$ \\
\hline $0 \cdot 990$ & $-0.097(61)$ & $11 \cdot 678(34)$ & 0.990 & $-0 \cdot 188(74)$ & $11 \cdot 685(21)$ \\
\hline \multicolumn{3}{|c|}{$X=0.3056$} & \multicolumn{3}{|c|}{$X=0.3981$} \\
\hline$\alpha$ & $N^{-1} \partial F^{\mathrm{ex}} / \partial \alpha$ & $\beta P \sigma^{3}$ & $\alpha$ & $N^{-1} \partial F^{\mathrm{ex}} / \partial \alpha$ & $\beta P \sigma^{3}$ \\
\hline $0 \cdot 850$ & $-4 \cdot 08(30)$ & $15 \cdot 47(17)$ & $0 \cdot 850$ & $-3 \cdot 83(44)$ & $15 \cdot 53(25)$ \\
\hline $0 \cdot 867$ & $-3 \cdot 89(32)$ & $14 \cdot 64(17)$ & 0.867 & $-3.97(22)$ & $14 \cdot 76(12)$ \\
\hline 0.883 & $-3 \cdot 58(14)$ & $13.902(64)$ & 0.883 & $-3.69(17)$ & $14.030(95)$ \\
\hline 0.900 & $-3 \cdot 07(13)$ & $13 \cdot 253(57)$ & 0.900 & $-3 \cdot 17(14)$ & $13 \cdot 350(61)$ \\
\hline 0.910 & $-2 \cdot 75(15)$ & $12 \cdot 930(56)$ & 0.910 & $-2 \cdot 79(13)$ & $12.977(57)$ \\
\hline $0 \cdot 925$ & $-2 \cdot 17(13)$ & $12 \cdot 493(50)$ & 0.925 & $-2 \cdot 30(13)$ & $12 \cdot 568(57)$ \\
\hline $0 \cdot 950$ & $-1.317(85)$ & $12 \cdot 016(42)$ & 0.950 & $-1 \cdot 44(11)$ & $12.031(37)$ \\
\hline 0.975 & $-0.621(73)$ & $11 \cdot 747(35)$ & 0.975 & $-0.655(81)$ & $11 \cdot 757(21)$ \\
\hline 0.990 & $-0.207(79)$ & $11 \cdot 685(33)$ & 0.990 & $-0 \cdot 26(10)$ & $11 \cdot 684(32)$ \\
\hline \multicolumn{3}{|c|}{$X=0.5000$} & \multicolumn{3}{|c|}{$X=0 \cdot 6019$} \\
\hline$\alpha$ & $N^{-1} \partial F^{\mathrm{ex}} / \partial \alpha$ & $\beta P \sigma^{3}$ & $\alpha$ & $N^{-1} \partial F^{\mathrm{ex}} / \partial \alpha$ & $\beta P \sigma^{3}$ \\
\hline $0 \cdot 850$ & $-3 \cdot 71(41)$ & $15 \cdot 3(3)$ & 0.850 & $-3 \cdot 33(27)$ & $14 \cdot 80(19)$ \\
\hline 0.867 & $-3 \cdot 71(23)$ & $14 \cdot 59(14)$ & $0 \cdot 867$ & $-3 \cdot 22(16)$ & $14 \cdot 15(11)$ \\
\hline $0 \cdot 883$ & $-3 \cdot 71(13)$ & $13.892(83)$ & 0.883 & $-2 \cdot 85(11)$ & $13 \cdot 545(73)$ \\
\hline $0 \cdot 900$ & $-2 \cdot 917(93)$ & $13 \cdot 247(43)$ & 0.900 & $-2 \cdot 56(11)$ & $13.064(58)$ \\
\hline 0.910 & $-2 \cdot 63(13)$ & $12 \cdot 945(82)$ & 0.910 & $-2 \cdot 305(86)$ & $12 \cdot 774(53)$ \\
\hline $0 \cdot 925$ & $-2 \cdot 170(90)$ & $12 \cdot 527(37)$ & 0.925 & $-1 \cdot 895(90)$ & $12 \cdot 431(52)$ \\
\hline $0 \cdot 950$ & $-1 \cdot 370(97)$ & $12 \cdot 035(43)$ & 0.950 & $-1 \cdot 272(64)$ & $11.932(35)$ \\
\hline 0.975 & $-0.713(75)$ & $11 \cdot 772(30)$ & 0.975 & $-0.607(78)$ & $11.755(28)$ \\
\hline $0 \cdot 990$ & $-0 \cdot 243(82)$ & $11 \cdot 678(26)$ & 0.990 & $-0 \cdot 249(76)$ & $11 \cdot 680(27)$ \\
\hline \multicolumn{3}{|c|}{$X=0.6944$} & \multicolumn{3}{|c|}{$X=0.7963$} \\
\hline$\alpha$ & $N^{-1} \partial F^{\mathrm{ex}} / \partial \alpha$ & $\beta P \sigma^{3}$ & $\alpha$ & $N^{-1} \partial F^{\mathrm{ex}} / \partial \alpha$ & $\beta P \sigma^{3}$ \\
\hline 0.850 & $-2 \cdot 74(14)$ & $14 \cdot 15(11)$ & $0 \cdot 850$ & $-1 \cdot 787(73)$ & $13 \cdot 268(54)$ \\
\hline 0.867 & $-2 \cdot 56(13)$ & $13 \cdot 649(97)$ & 0.867 & $-1 \cdot 666(70)$ & $12 \cdot 967(55)$ \\
\hline \multirow[t]{2}{*}{0.883} & $-2 \cdot 285(94)$ & $13 \cdot 167(65)$ & 0.883 & $-1.534(67)$ & $12 \cdot 680(40)$ \\
\hline & & & 0.900 & $-1 \cdot 360(73)$ & $12 \cdot 439(39)$ \\
\hline 0.910 & $-1 \cdot 819(69)$ & $12 \cdot 577(42)$ & 0.910 & $-1.274(72)$ & $12 \cdot 292(42)$ \\
\hline 0.925 & $-1 \cdot 537(72)$ & $12 \cdot 283(42)$ & 0.925 & $-1 \cdot 067(63)$ & $12 \cdot 114(39)$ \\
\hline 0.950 & $-1.099(59)$ & $11.963(29)$ & 0.950 & $-0.750(53)$ & $11 \cdot 878(27)$ \\
\hline 0.975 & $-0.527(69)$ & $11 \cdot 746(29)$ & 0.975 & $-0.378(66)$ & $14.568(33)$ \\
\hline 0.990 & $-0 \cdot 240(64)$ & $11 \cdot 684(29)$ & 0.990 & $-0 \cdot 161(74)$ & $11.690(31)$ \\
\hline
\end{tabular}




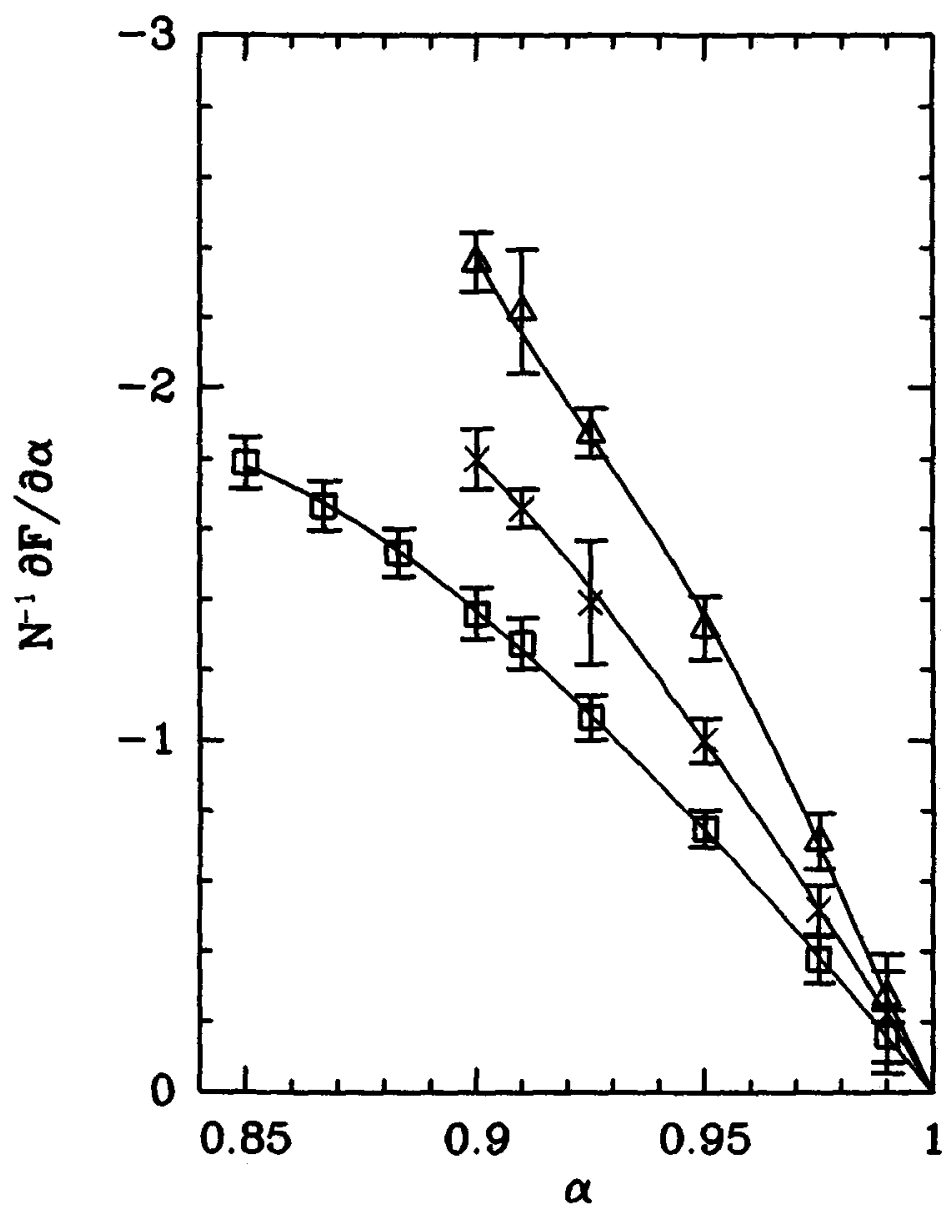

Figure 1. The derivative of the Helmholtz free energy per particle as a function of the diameter ratio $\alpha$ for a mole fraction of large spheres $X=0.7963$ for a substitutional disordered FCC crystal. Results for three differenct packing fractions are shown: $\phi=0.545(\square), 0.560(x)$ and $0.576(\Delta)$. The full lines are least square fits by polynomials of degree four.

In figures 1 and 2 we have plotted the derivatives versus the diameter ratio for three different packing fractions at two quite different compositions. The figures show clearly that the absolute value of the derivative increases with increasing packing fraction at constant diameter ratio $\alpha$. Also notable is the qualitative difference in the dependence on $\alpha$ near the monodisperse limit for mole fractions $X=0.2037$ and 0.7963 : for $X=0.2037$ the derivative is a concave function of $\alpha$, whereas for $X=0.7963$ it is a convex function. Furthermore, the absolute value of the derivative tends to go to a maximum for $X=0.2037$ at a packing fraction of $\phi=0.545$ near $\alpha=0 \cdot 85$.

To obtain the difference in free energy between the solid mixture and the monodisperse solid, we fitted a polynomial in $X$ to the data points and determined $\Delta F^{\text {ex }}$ by integration. Results are shown in table 5 . The free energy differences can be estimated with an accuracy better than $10 \%$. The free energy per particle of the monodisperse solid is around 6 (in units of $k_{\mathrm{B}} T$ ) for these packing fractions [3], so the free energy differences are a quite small addition. Because the results of Frenkel and Ladd are 




Figure 2. The derivative of the Helmholtz free energy per particle as a function of the diameter ratio $\alpha$ for a mole fraction of large spheres $X=0.2037$ for a substitutional disordered FCC crystal. Results for three differenct packing fractions are shown: $\phi=0.545(\square), 0.560(\mathrm{x})$ and $0.576(\Delta)$. The full lines are least square fits by polynomials of degree four.

very accurate, the error in the total free energy of a solid mixture will be fully determined by the error in the free energy difference, except for $\alpha=0.95$. Furthermore, a small size dependence of at most $1 \%$ was found for the free energy of a monodisperse FCC or HCP lattice, when going from 32 to 1152 particles. It is clear from table 5 that such a small effect will be totally masked by the large absolute error in the difference of the free energy.

When tables 3 and 5 are compared with each other, we find good agreement between methods I and II: the differences between the methods are always less than two standard deviations of the results of method II. In the present example the particle swapping yields lower estimates of free energy differences than the thermodynamic integration method (II). This is, however, not always the case.

We have also applied method II to a substitutionally ordered crystal. In particular, we have calculated the free energy derivative with respect to diameter ratio for a $1: 1$ ordered lattice with a caesium chloride structure. Results are given in table 6. Again, MD was used to average over configuration space, but this time without particle 
Table 5. Results of integrating the derivative with respect to the diameter ratio $\alpha$ at constant packing fraction and composition: free energy differences between a solid mixture of mole fraction $X$ at a diameter ratio $\alpha$ and the monodisperse solid. These differences were obtained by integrating the derivative of the free energy with respect to the diameter ratio at constant mole fraction. For $\alpha=0.85$ the integration was performed at a packing fraction of $\phi=0.545$, for $\alpha=0.90$ at $\phi=0.560$ and for $\alpha=0.95$ at $\phi=0.576$.

\begin{tabular}{|c|c|c|c|c|c|}
\hline \multicolumn{2}{|c|}{$\alpha=0.95$} & \multicolumn{2}{|c|}{$\alpha=0.90$} & \multicolumn{2}{|c|}{$\alpha=0.85$} \\
\hline$X$ & $\Delta\left(\beta F^{\mathrm{ex}}\right) / N$ & $X$ & $\Delta\left(\beta F^{\mathrm{ex}}\right) / N$ & $X$ & $\Delta\left(\beta F^{\mathrm{ex}}\right) / N$ \\
\hline $0 \cdot 2037$ & $0.04589(20)$ & 0.2037 & $0.157(11)$ & $0 \cdot 2037$ & $0.279(23)$ \\
\hline 0.3981 & $0.06126(17)$ & $0 \cdot 3981$ & $0.197(12)$ & $0 \cdot 3981$ & $0.335(29)$ \\
\hline 0.5000 & - & 0.5000 & $0.192(12)$ & 0.5000 & $0.321(25)$ \\
\hline 0.6019 & $0.05536(18)$ & 0.6019 & $0 \cdot 1688(99)$ & 0.6019 & $0.277(20)$ \\
\hline 0.7983 & $0.03411(16)$ & 0.7983 & $0.097(13)$ & 0.7983 & $0 \cdot 152(13)$ \\
\hline
\end{tabular}

swapping moves. In contrast with the results for the disordered crystals, the derivatives of the free energy are positive. There is a maximum around $\alpha=0.775$, and for the monodisperse limit the derivative tends to go to zero again, as expected. The results for $\alpha=0.975$ and 0.900 are not reliable: in the monodisperse limit the $\mathrm{CsCl}$ structure transforms into a BCC structure, which is mechanically unstable [10]. However, in MD simulations of a system of 108 particles we found that the crystal becomes mechanically unstable only below a packing fraction $\phi=0.542$ $\left(\rho^{*}=1.035\right)$. Hence the results in table 6 for $\phi=0.576$ are not affected. The relatively large errors in the pressure for $\alpha$ close to 1 may be a remnant of the presence of a mechanical instability in larger systems.

Generalizing the method to multicomponent hard sphere mixtures or mixtures of non-spherical hard particles should be relatively straightforward, since it requires no qualitative changes of the method.

Table 6. $\mathrm{CsCl}$ structure. The derivative of the free energy with respect to the diameter ratio per particle $N^{-1} \partial F^{\text {ex }} / \partial \alpha$ and the pressure $\beta P \sigma^{3}$ are represented as functions of the diameter ratio $\alpha$ at a packing fraction $\phi=0.576$.

\begin{tabular}{|c|c|c|}
\hline$\alpha$ & $N^{-1} \partial F^{e x} / \partial \alpha$ & $\beta P \sigma^{3}$ \\
\hline 0.600 & $-0.52(13)$ & $18 \cdot 211(74)$ \\
\hline 0.650 & $0.255(77)$ & $16 \cdot 116(47)$ \\
\hline 0.700 & $1 \cdot 284(63)$ & $15.472(22)$ \\
\hline 0.725 & $1.723(80)$ & $15.575(25)$ \\
\hline 0.750 & $1.972(77)$ & $15.907(25)$ \\
\hline 0.775 & $2 \cdot 016(82)$ & $16.401(24)$ \\
\hline 0.800 & $1.85(11)$ & $16.974(37)$ \\
\hline 0.825 & $1 \cdot 51(10)$ & $17 \cdot 510(59)$ \\
\hline 0.850 & $1.070(82)$ & $17 \cdot 930(54)$ \\
\hline $0 \cdot 867$ & $0.84(10)$ & $18 \cdot 154(73)$ \\
\hline 0.883 & $0.591(82)$ & $18 \cdot 315(62)$ \\
\hline 0.900 & $0.40(11)$ & $18 \cdot 396(74)$ \\
\hline 0.925 & $0.225(93)$ & $18 \cdot 432(72)$ \\
\hline 0.950 & $0.08(11)$ & $18.437(82)$ \\
\hline 0.975 & $-0.41(22)$ & $18 \cdot 62(21)$ \\
\hline 0.990 & $-0.33(22)$ & $18 \cdot 77(22)$ \\
\hline
\end{tabular}




\section{Summary and conclusions}

Here we summarize the relative merits of the particle swapping and thermodynamical integration methods.

The swapping method, in which a reversible path is constructed by varying the composition has the following advantages.

(i) It is simple to use if the overlap criterion is known. As this criterion is already needed in every $\mathrm{MC}$ algorithm, the implementation of the particle swapping method in existing programs is easy.

(ii) The method does not consume much extra CPU time.

(iii) The method can be used both in MD and MC.

However, despite these advantages, the method has a number of serious drawbacks.

(i) It only gives reliable estimates if the difference in the size or shape of the unlike particles is rather small.

(ii) Because we only swap one particle at a time in each simulation, a large number of different simulations are required to cover the whole range of mole fractions between 0 and 1 for a system of $N$ particles. This makes the method cumbersome even for small systems. Furthermore, summation of the free energy differences can lead to large statistical errors. In practice, one is forced to use rather small systems. Therefore the results may suffer from a large size dependence.

(iii) The method cannot be applied directly to ordered crystals.

(iv) The method is less suitable for mixtures with more than two components.

The second method, based on the derivative of the free energy with respect to the diameter ratio, does not suffer from these drawbacks. The advantages of this method can be summarized as follows.

(i) One needs the same quantities that are needed to calculate the pressure. Hence, again, implementing this method is easy and cheap.

(ii) The method has a wide range of applicability: mixtures of particles that differ appreciably in form or size can be treated easily. It can be applied at any density and it is suitable for liquids as well as disordered and ordered solids.

(iii) It can easily be generalized to other systems.

However, the method also has shortcomings.

(i) Owing to the fact that the relative error in the derivative of the free energy may be large (larger than the relative error of the pressure), long runs are necessary.

Comparing the relative merits of both methods, it is clear that the thermodynamic integration method is preferable to the method of interchanging particles. However, as an alternative method the swapping method can be very useful to get a rough estimate of the free energy differences. It can therefore be used as a check of the results of method II.

The investigations reported in this paper were supported in part by the Netherlands Foundations for Chemical Research (SON) with financial aid from the Netherlands Organization for Scientific Research (NWO). The work of the FOM Institute 
is part of the scientific programme of FOM and is supported by the Netherlands Organization for Scientific Research (NWO).

\section{References}

[1] McDonald, I. R., 1972, Molec. Phys., 24, 391.

[2] Hoover, W. G., and ReE, F. M., 1967, J. chem. Phys., 49, 3609.

[3] Frenkel, D. and Ladd, A. J. C., 1984, J. chem. Phys., 81, 3188.

[4] PRigogine, I., and Defay, R., 1954, Chemical Thermodynamics (Longmans Green), Chap. 20; Guggenheim, E. A., 1986, Thermodynamics (North-Holland), Chap. 4.

[5] Kranedonk, W. G. T., and Frenkel, D., 1991, Molec. Phys., 72, 679.

[6] WIDOM, B., 1963, J. chem. Phys., 39, 2808.

[7] Shing, K. S., 1985, Chem. Phys. Lett., 119, 149; 1986, J. chem. Phys., 85, 4633.

[8] Sindzingre, P., Massobrio, C., Ciccotti, G., and Frenkel, D., 1987, Chem. Phys. Lett., 136, 35.

[9] BenNetT, C. H., 1976, J. comput, Phys., 22, 245.

[10] Hoover, W. G., Young, D. A., and Grover, R., 1972, J. chem. Phys., 56, 2207. 